MI-mAbs,

démonstrateur

préindustriel

soutenu par

le programme

d'investissements

La mission du démonstrateur industriel MI-mAbs, porté par Aix Marseille Université avec le CNRS, I'Inserm, l'institut Paoli Calmettes, Sanofi et Innate pharma dans la gouvernance, est de proposer un service de validation précoce de cibles thérapeutiques, adressables par anticorps monoclonaux, pour l'immuno-modulation en cancérologie et dans les maladies inflammatoires. Elle consiste à générer, pour une cible donnée, des candidats anticorps compatibles avec un développement pharmaceutique ultérieur, ainsi qu'une preuve de concept de l'activité pharmacologique, in vitro sur des cellules ou des tissus de donneurs sains et de patients, et in vivo dans des modèles pharmacologiques adaptés, utilisant notamment des souris génétiquement modifiées. Le travail pour une cible donnée dure environ 2 ans, et la structure Ml-mAbs à pleine charge doit pouvoir travailler sur 6 à 8 cibles en parallèle ( 3 à 5 actuellement). La structure maîtrise un ensemble de technologies clés dans l'ingénierie des anticorps (humanisation, anticorps couplés à des toxines [3], anticorps bispécifique [1]) et leur étude pharmacologique, permettant de qualifier des candidats médicaments pour un développement pharmaceutique proprement dit.

Le marché des anticorps monoclonaux thérapeutiques est estimé à 90 milliards d'euros par an et augmente chaque année de plus de $10 \%$, avec une activité de recherche \& développement représentant au moins $10 \%$ de cette somme. La France est pour le moment peu présente le long de la chaîne de valeur, hormis Sanofi et quelques biotechs du type Innate Pharma. II est bien difficile de trouver un anticorps issu de la recherche française, pourtant très active dans ces domaines, qui ait pu aboutir à un produit commercial. MI-mAbs complète la structuration de la filière anticorps en France, impulsée par les pouvoirs publics avec le Labex MAbImprove et la structure MabDesign.

La structure MI-mAbs doit à moyen terme fournir un service décisif : 1) aux structures de valorisation de la recherche publique (SATT ${ }^{1}$, Inserm-Transfert, etc.) pour générer et qualifier des candidats médicaments valorisables auprès des industriels, 2) aux start-up du domaine n'ayant pas les moyens techniques nécessaires

PIA : programme d'investissement d'avenir.

${ }^{1}$ Sociétés d'accélération du transfert de technologies.

d'Avenir pour la validation précoce de cibles thérapeutiques à l'aide d'anticorps monoclonaux

\section{François Romagné}

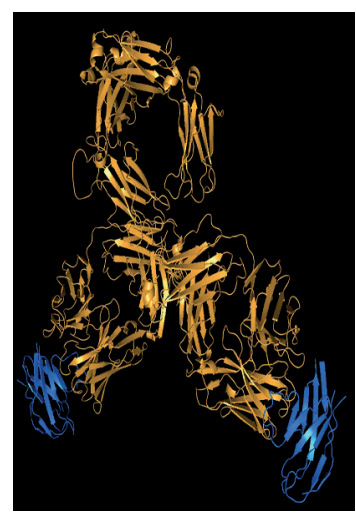

Université Aix Marseille, 117 avenue de Lattre de Tassigny, 13009 Marseille, France.

francois.romagne@univ-amu.fr

pour effectuer le travail de validation, 3) aux industries de biotechnologies et pharmaceutiques désirant un service professionnel dans ce domaine en externalisant leur qualification amont, permettant le passage à un développement pharmaceutique.

MI-mAbs est maintenant bien établie : elle a finalisé un recrutement de haut niveau (33 personnes, dont 9 doctorants, issues de l'industrie ou de la recherche académique) et la plateforme est maintenant totalement structurée, depuis mi-2017, dans un bâtiment propre, en accord avec le projet initialement déposé, pour proposer un service original de validation de cibles précoces à l'aide d'anticorps monoclonaux. La capacité de la structure à délivrer des résultats de validation de cibles, bien que limitée au début du projet dans des laboratoires d'accueil, a été testée et avérée au travers de nos premiers contrats de recherche avec nos industriels partenaires (Innate Pharma et Sanofi). Notamment des anticorps anti-CD73, anti-CD39 [2] et antiSiglec (sialic acid-binding immunoglobulin-type lectins) ont été générés et caractérisés en collaboration avec Innate Pharma et ont été qualifiés pour le développement pharmaceutique proprement dit. Un 
nouveau format d'anticorps a, par ailleurs, été validé en collaboration avec Sanofi [1] et devrait entrer en développement sous peu.

Notre installation dans nos laboratoires propres et le recrutement correspondant permettent maintenant d'augmenter notre activité et de nous ouvrir à de nouveaux clients et partenaires. D'ores et déjà, nous avons passé des contrats en 2017 et 2018 avec des start-up françaises (Talix, Biomunex, Diaccurate, Igyxos), les SATT Sud-Est et Sud-Ouest, ainsi que de nouveaux industriels (Servier, Beckman Coulter, HalioDx et Cisbio) qui nous confient la validation expérimentale de leurs cibles ou du travail d'ingénierie des anticorps.

Nous avons déjà généré un montant de contrats significatifs (3 millions d'euros de revenus de contrats cumulés entre 2014 et 2017). Pour 2018, le montant des revenus de contrat a atteint 2 millions d'euros (1,1 million en 2017) pour une dépense annuelle de 3,5 millions hors investissement. MI-mAbs peut maintenant augmenter son activité pour tendre vers l'autosuffisance. Nous structurons nos activités commerciales, avec notamment un personnel de business développement dédié. L'activité des industries pharmaceutiques et biotechnologiques dans le domaine est très importante: ce seront nos cibles prioritaires avec l'aide au développement de start-up et un travail conjoint avec les acteurs de la valorisation de la recherche. $\diamond$

MI-mAbs, pre-industrial demonstrator supported by PIA for the early validation of therapeutic targets using monoclonal antibodies
LIENS D'INTÉRÊT

L'auteur déclare n'avoir aucun lien d'intérêt concernant les données publiées dans cet article.

\section{RÉFÉRENCES}

1. Gauthier L, Morel A, Anceriz N, et al. Multifunctional natural killer cell engagers targeting NKp46 trigger protective tumor immunity. Cell 2019; 177 : 1701-13-el6.

2. Perrot I, Michaud HA, Giraudon-Paoli M, et al. Blocking antibodies targeting the CD39/CD73 immunosuppressive pathway unleash immune responses in combination cancer therapies. Cell Rep $2019 ; 27: 2411-25$-e9.

3. Lhospice F, Bregeon D, Belmant C, et al. Site-specific conjugation of monomethyl auristatin $\varepsilon$ to anti-CD30 antibodies improves their pharmacokinetics and therapeutic index in rodent models. Mol Pharm 2015; 12 : 1863-71

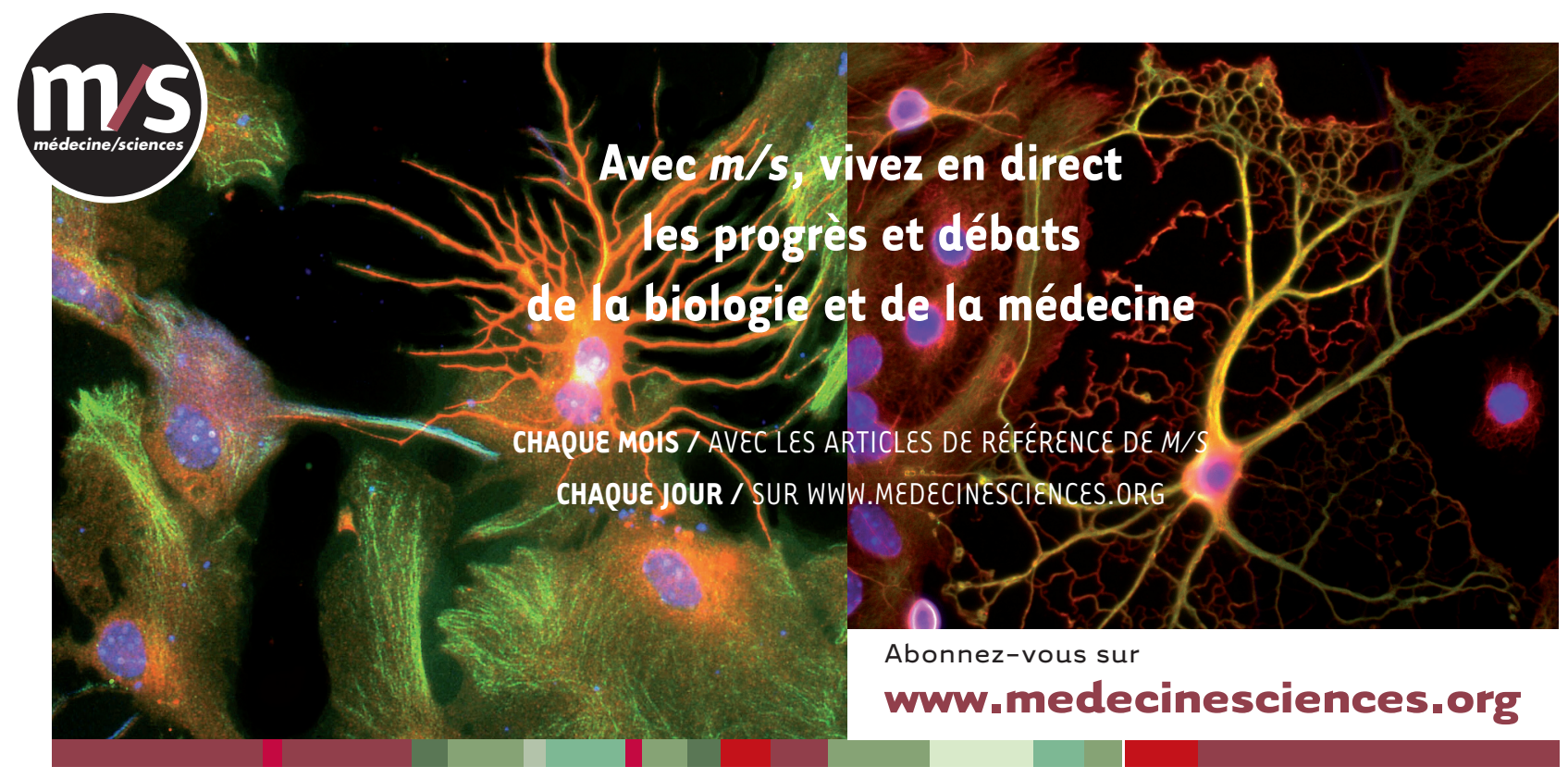

TIRÉS À PART

F. Romagné

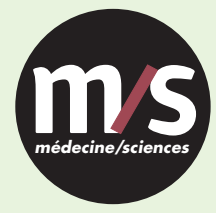

Tarifs d'abonnement $\mathrm{m} / \mathrm{s}-2019$

Abonnez-vous

à médecine/sciences
$>$ Grâce à $m / s$, vivez en direct les progrès des sciences biologiques et médicales

Bulletin d'abonnement page 1230 dans ce numéro de $\mathrm{m} / \mathrm{s}$

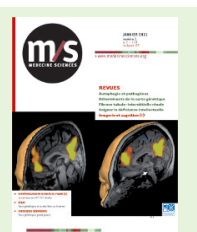

